\title{
Evaluation of accuracy of complete-arch multiple-unit abutment-level dental implant impressions using different impression and splinting materials.
}

\author{
Type: Article
}

Abstract:

Purpose: This in vitro study evaluated the accuracy of multiple-unit dental implant casts obtained from splinted or nonsplinted direct impression techniques using various splinting materials by comparing the casts to the reference models. The effect of two different impression materials on the accuracy of the implant casts was also evaluated for abutment-level impressions. Materials and Methods: A reference model with six internal-connection implant replicas placed in the completely edentulous mandibular arch and connected to multi-base abutments was fabricated from heat-curing acrylic resin. Forty impressions of the reference model were made, 20 each with polyether (PE) and polyvinylsiloxane (PVS) impression materials using the open tray technique. The PE and PVS groups were further subdivided into four subgroups of five each on the bases of splinting type: no splinting, bite registration PE, bite registration addition silicone, or autopolymerizing acrylic resin. The positional accuracy of the implant replica heads was measured on the poured casts using a coordinate measuring machine to assess linear differences in interimplant distances in all three axes. The collected data (linear and three-dimensional [3D] displacement values) were compared with the measurements calculated on the reference resin model and analyzed with nonparametric tests (Kruskal-Wallis and Mann-Whitney). Results: No significant differences were found between the various splinting groups for both PE and PVS impression materials in terms of linear and 3D distortions. However, small but significant differences were found between the two impression materials (PVS, $91 \mathrm{mu} \mathrm{m}$; PE, $103 \mathrm{mu} \mathrm{m}$ ) in terms of 3D discrepancies, irrespective of the splinting technique employed. Conclusions: Casts obtained from both impression materials exhibited differences from the reference model. The impression material influenced impression inaccuracy more than the splinting material for multiple-unit abutment-level impressions.

\begin{tabular}{|c|l|}
\hline Author & Buzayan, M.; Baig, M. R.; Yunus, N. \\
\hline Source & International Journal of Oral \& Maxillofacial Implants \\
\hline ISSN & $0882-2786$ \\
\hline DOI & - \\
\hline Volume (Issue) & $28(6)$ \\
\hline Page & $1512-1520$ \\
\hline Year & 2013 \\
\hline
\end{tabular}

Keyword:

EDENTULOUS PATIENTS; PROSTHESES; FIT; dental implants; dental impression technique; dental models; impression accuracy

URL:

- $\quad$ http://apps.webofknowledge.com search via Accession No >> 000328125900033

- Full text available at : http://www.ncbi.nlm.nih.gov/pubmed/24278919 